\title{
Are periods of feeding - fasting protective during critical illness?
}

Zudin Puthucheary ${ }^{1,2}$ and Jan Gunst ${ }^{3}$

${ }^{1}$ William Harvey Research Institute, Barts and The London School of Medicine and Dentistry, Queen Mary University of London, London, UK; ${ }^{2}$ Adult Critical Care Unit, Royal London Hospital, London, UK; ${ }^{3}$ Clinical Department and Laboratory of Intensive Care Medicine, Department of Cellular and Molecular Medicine, KU Leuven, Leuven, Belgium

\section{Corresponding author}

Dr Zudin Puthucheary, Critical Care and Perioperative Medicine Research Group, Adult Critical Care Unit, Royal London Hospital, London, E1 1BB, United Kingdom

Email: z.puthucheary@qmul.ac.uk

Tel: +44(0) 2035940351

Jan Gunst, Clinical Department and Laboratory of Intensive Care Medicine,

Department of Cellular and Molecular Medicine, KU Leuven, Leuven, Belgium.

Email: jan.gunst@kuleuven.be

Word Count:2078/2500 
Abstract (200 words): 192/200 words

\section{Purpose of review}

To review the mechanisms how intermittent feeding regimens could be beneficial in critically ill patients.

\section{Recent findings}

Large randomised controlled trials have failed to demonstrate consistent benefit of early, enhanced nutritional support to critically ill patients, and some trials even found potential harm. Although speculative, the absence of a clear benefit could be explained by the continuous mode of feeding in these trials, since data in health suggest plausible mechanistic effects that intermittent feeding could be as effective or superior to continuous feeding in the critically ill. Potential protective mechanisms include avoidance of the muscle-full effect and improved protein synthesis, improved insulin sensitivity, better preservation of circadian rhythm, and fastinginduced stimulation of autophagy and ketogenesis. Randomised controlled trials comparing continuous versus intermittent feeding regimens in critically ill patients have shown mixed results, albeit with different design and inclusion of relatively few patients. In all studies, the fasting interval was relatively short (4-6 hours maximum), which may be insufficient to develop a full fasting response and associated benefits.

\section{Summary}

These findings open perspectives for the design and clinical validation of intermittent feeding regimens for critically ill patients. The optimal mode and duration of the fasting interval, if any, remain unclear.

Key words: critical illness, intermittent feeding, fasting, autophagy, ketones 


\section{Background}

Despite the association of underfeeding with impaired outcome of critically ill patients, recent large randomised controlled trials (RCTs) have not shown clear benefit by early full nutritional support, and some RCTs even suggested potential harm by such approach[1-6]. However, in these RCTs, artificial feeding was administered continuously, and increasing evidence from studies in healthy individuals suggests that intermittent feeding regimens, whereby feeding periods are alternated by fasting intervals, may be superior to continuous feeding. Indeed, numerous animal studies and several (pre)clinical studies have shown that intermittent fasting protected against age-related diseases through promoting cellular stress resistance by various mechanisms, and recent evidence has implicated these pathways as potentially important in recovery from critical illness[7] ${ }^{* *}[8,9]$. Moreover, mechanistic studies have suggested that intermittent delivery of nutrients may have additional benefit through other mechanisms[10].

Three modes of discontinuous feeding have been reported: cyclical, intermittent and bolus feeding. Cyclical feeding alternates one fasting and feeding interval per day. In intermittent and bolus feeding, feeding is provided 4-6 times a day and administered over 20-60 min (intermittent feeding) or over 5-10 min (bolus feeding)[10]. In the literature, the term intermittent feeding has also been used to cover other forms of discontinuous feeding.

We here summarize the most recent evidence regarding intermittent feeding regimens in critically ill patients, focusing on the potential protective mechanisms involved.

\section{Mechanisms of how intermittent feeding regimens could be beneficial}

\section{Avoidance of the muscle-full effect}

Continuous amino acid delivery does not lead to continued muscle protein synthesis. After 2-3 hours, myofibrillar synthetic rates return to baseline, before decreasing further[11]. This is true both for enteral and parenteral routes of delivery, and is termed the "muscle full-effect". The mechanism that has been proposed is the inability of skeletal muscle to continuously absorb amino acids, with the rate-limiting step being the protein synthetic apparatus[11].

However, a recent multicenter single blinded RCT of bolus versus continuous feeding found no difference between groups in ultrasonographic loss of muscle mass at 10 days (the primary outcome measure) in mechanically ventilated patients with multiorgan failure[12]**. This was carried out in eight intensive care units in the UK, and 127 patients enrolled, with 62 patients allocated to a 6 4-hourly feed during 24 hours. No difference in muscle mass between groups was detected in the perprotocol sub analysis. While intermittent/bolus feeding has been shown to overcome the muscle-full effect in healthy volunteers, as a sole intervention it may not be able to overcome adverse stimuli such as immobilization or inflammation in the critically ill[13, 14]. Alternatively, the median duration of the intervention -4 days- may have been too short to detect a benefit, as in many nutritional trials, and the number of patients may have been too small[15]. 
Continuous feeding increases insulin resistance by inhibition of hepatic glucose transport, a process already compromised in the critically ill[16].. In the second week of critical illness, patients fed intermittently required less exogenous insulin than those fed continuously[12]**. In the 63 patients in the per-protocol analysis, mean daily insulin delivered was higher on day 8 of critical illness in the continous arm (89 (95\% Cl $41-111)$ vs. $45(95 \% \mathrm{Cl} 24-65)$ and continued to be so on days 9 and $10 . "$ This may, in the future, be the preferential mode of nutrition delivery for those patients with persistent critical illness, for whom recovery of normal metabolic function could improve muscle mass, which needs further investigation.

When tested prospectively, bolus feeding did not result in a higher event rate of hypoglycaemia, though glucose variability increased (Coefficient of variation 17.8 $(95 \% \mathrm{Cl} 18.6-20.4)$ vs. $13.0 \quad(95 \% \mathrm{Cl} 14.0-15.7))$ especially amongst diabetic individuals[12]**. In the acute phase, if intermittent/bolus feeding is to be used, more frequent glucose measurements and insulin titration may be required than is often performed as increasing glycaemic variability has been associated with poorer outcomes in critically ill patients with and without diabetes.

\section{Preservation of circadian rhythm}

Lipid and protein and energy homeostasis are affected in some degree by the circadian rhythm. This central clock regulation in turn regulates local clocks responsible for metabolism and cellular processes in peripheral tissues, including skeletal muscle. Specific to altered physiology and muscle wasting in the critically ill patient, the hepatic clock regulates glycaemic control and glucose clearance, the pancreatic clock regulates insulin secretion and sensitivity and the skeletal muscle clock regulates glucose uptake and metabolism[17]. Adaptation to novel feeding patterns (e.g. continuous feeding while critically ill) is not immediate, taking from 3 days (liver) to 7 days (cardiac) with adaptation times of skeletal muscle being unknown[18].

Circadian misalignment is detrimental to metabolism. Continuous nutritional delivery alters circadian rhythms of intestinal hormones, and post-prandial rhythms of ghrelin and insulin release in response to nutrition[19]. Speculatively, these patterns of altered physiology might be avoided by preventing reprogramming of the circadian rhythm using intermittent feeding regimens. One could then hypothesise that this would then promote better sleep quality in the critically ill patient, which is crucial for successful rehabilitation.

\section{Nutritional delivery}

International audits repeatedly demonstrated actual deliveries of nutritional requirements to be close to $60 \%$ of daily requirements [20]. Barriers to adequate nutritional delivery include prolonged fasting for procedures (e.g. central line placement, surgical or radiological procedures), premature feed cessation based on gastric residual volumes, and failure to start or increase feeding regimes appropriately. After critical illness, underfeeding continues due to patient (muscle weakness and dysphagia) and healthcare delivery (feeding tube removal, lack of structured meal times) factors[21].. A per-protocol analysis of a phase II trial suggested patients achieved their nutritional targets more frequently (OR 1.52 $(95 \% \mathrm{Cl} 1.16-1.99)$ for $80 \%$ delivery of target protein and OR 1.59 (95\% Cl1.21-2.08) 
for target energy) with a bolus feeding regime, addressing the many systems barriers to nutrition delivery in a similar fashion to volume based feeding [12]**. This benefit may be context-specific, as patients who are more stable may derive less benefit[22]. Despite this, bolus feeding is increasingly common in the community, especially among the more active patient[23].

The impact of intermittent/bolus feeding on enteral feeding intolerance has been a subject of contention. While such feeding strategy may seem more physiological, the current methodology of measuring enteral feeding intolerance (gastric residual volume) is highly imprecise, and potentially correlates poorly with actual intolerance. Additionally, the relationship with diarrhoea is unclear, as a result of a high background event rate[24]. Interestingly, a prospective multi-centre RCT found no differences in enteral feed tolerance between continuous versus bolus feeding[12 $]^{* *}$.

\section{Timing and synergy with exercise}

In health, the combination of exercise and amino acids results in an increase in muscle protein synthesis, increasing muscle mass. With increasing age, not only does the response to exercise attenuate, but also the window to respond to the combined stimulus decreases[25]. Intermittent feeding therefore may allow the rehabilitation team to time nutrition delivery with exercise, maximizing the efficiency of the interventions to improve muscle mass and function.

\section{Intermittent feeding as part of the recovery process}

A recent systematic review concluded that rehabilitation interventions in the critically ill did not consistently translate into long-term functional benefit[26]*. In health, and among healthcare professionals, attitudes and behaviours to own exercise and health priorities are given to adequate sleep (both quality and quantity) and adequate nutrition (both protein and energy) as part and parcel of the exercise and fitness process. Curiously, in clinical trials, these components have been tested as individual strategies. Although speculative, intermittent feeding may be a method of nutritional delivery that enhances the rehabilitation process (increases nutritional delivery, decreases muscle-full effect), normalises metabolism (decreases insulin resistance) and increases sleep quality, especially when combined with exercise.

\section{Potential mechanisms why intermittent fasting may be beneficial}

Apart from potential benefit through intermittently delivering nutrients, several hypothesised benefits of intermittent feeding regimens are specifically mediated by the response to intermittent fasting.

\section{Fasting-induced autophagy}

A potential cell-protective effect induced by fasting is activation of autophagy[27]. In this recovery process, which is normally activated by cellular stressors and subsequent cellular damage, intracellular debris is recruited to an autophagosomal vesicle, followed by fusion with lysosomes and degradation. However, the process is strongly inhibited by nutrients and insulin, which may lead to accumulation of cellular damage[8]. Of note, autophagy is the only repair mechanism able to clear macromolecular damage, including damaged organelles and potentially toxic protein 
aggregates. Hence, in cases of insufficient autophagy, other damage repair and/or catabolic pathways cannot fully compensate for a lack of autophagic activity[8]. Clinical studies have shown hallmarks of insufficient autophagy in liver of prolonged critically ill patients who received full feeding[28]. Moreover, in a secondary analysis of the EPaNIC study, early parenteral nutrition suppressed autophagy in muscle, which correlated with more weakness[29]. The crucial role of autophagy in mediating recovery in critically ill states has been confirmed by several animal models that genetically and pharmacologically interfered with autophagy[8, 30]. Pharmacological autophagy activation in humans is cumbersome, however, since no specific autophagy activators are available[31]. Hence, physiological activation of autophagy through intermittent fasting or fasting-mimicking diets may be more straightforward. Interestingly, in other models, animal studies have shown that fasting-mimicking diets are able to protect against age-related disease in part by activating autophagy $[32,33]$. The duration of the fasting interval needed to activate autophagy in critically ill patients remains unclear[34]*

\section{Enhanced ketogenesis}

A second, emerging mechanism by which fasting may be protective in critical illness is enhanced ketogenesis. Indeed, activation of ketogenesis is an essential part of the human fasting response, and increasing evidence suggests that enhanced ketogenesis may be beneficial in critical illness[35, 36]. Apart from being more energy-efficient than glucose as substrate, ketones have signalling properties[35]. Ketone supplementation may stimulate muscle regeneration, and improved exercise endurance in athletes[37, 38]. In animal models of brain injury and sepsis, ketone supplementation and/or a ketogenic diet protected against brain injury and muscle weakness, respectively[39, 40]. In addition, ketones may exert anti-inflammatory effects[41]. In critically ill children, withholding early parenteral nutrition activated ketogenesis up to the millimolar range, which statistically mediated part of its clinical benefit[36]. Finally, enhanced ketogenesis may explain the obesity paradox, i.e. the observation that mild overweight/obesity may be protective in critical illness [42].

\section{Other mechanisms}

Apart from activating autophagy and ketogenesis, fasting also enhances the activity and/or expression of various molecules that had cell-protective effects in other settings, including peroxisome proliferator-activated receptor $\gamma$ coactivator $1 \alpha$ (PGC$1 \alpha)$, sirtuins, and fibroblast growth factor $21[7]^{* *}$ [35]. These signalling molecules and enzymes may modulate mitochondrial biogenesis and the unfolded protein response, which are potentially important repair pathways in critical illness[9]. Whether these pathways are affected by intermittent fasting in critically ill patients, remains to be investigated, however.

Finally, intermittent fasting may be beneficial through intermittent restoration of gastric acidity, which may inhibit bacterial colonization and prevent nosocomial pneumonia. A small RCT ( $n=60)$ has confirmed temporary lowering of intragastric $\mathrm{pH}$ by cyclical feeding as compared to continuous feeding, albeit without impact on colonization or incidence of pneumonia[43]. Nevertheless, the study may lack power to detect a meaningful benefit. 
Clinical evidence and duration of the fasting interval

Despite the physiological rationale and evidence in other models, clinical evidence favouring discontinuous feeding and intermittent fasting in critically ill patients is scarce. In a recent review, 12 RCTs were identified, with substantial heterogeneity in design and reported endpoints, and divergent clinical results[44]** Recently, a RCT did not find clinical benefit by bolus feeding as compared to continuous feeding[12]**. All RCTs until now included relatively few participants, however. Therefore, neutral results may be explained by a lack of power. Moreover, in these RCTs, feeding was typically administered every 4-6 hours, whereby the fasting interval may have been too short to activate potential cell-protective pathways [12, $44]^{* *}$. Indeed, the ideal duration of the fasting interval in critically ill patients, if any, remains unclear. In a recent pilot study, Van Dyck et al. found increased ketogenesis after 4 hours of fasting, and alterations in other markers of the fasting response after 12 hours of fasting[34]*. However, markers of autophagy in white blood cells were not affected. This may mean that 12 hours fasting is not sufficient to develop a full metabolic fasting response, although white blood cells may not be a good readout for autophagy at the tissue level[34]*. With prolonged fasting intervals, however, there may be a higher chance of feeding intolerance, since more feeding would have to be administered over a shorter time period if underfeeding is to be avoided. In this regard, a systematic review found -with shorter fasting intervals- more diarrhoea with intermittent/bolus feeding[45]*. However, the systematic review only identified 5 RCTs, and other RCTs have been neutral with regard to feeding intolerance, including the most recent multi-centre RCT $[12,44]^{* *}$.

\section{Conclusion}

Data from preclinical studies and those conducted in health provide mechanistic plausibility that alternating feeding and fasting periods in critically ill patients may be beneficial. Potential protective mechanisms include avoidance of the muscle-full effect, improvement of circadian rhythm and insulin sensitivity, and fasting-induced activation of autophagy and ketogenesis, among others. Despite clear rationale and promising mechanistic studies, clinical evidence remains scarce. Existing RCTs included relatively few patients and have not shown patient-centred benefit by intermittent feeding regimens. This may be explained by a lack of statistical power to detect a clinical benefit (or definitive lack of benefit or indeed harm), or by the relatively short duration of the fasting interval. The ideal intermittent feeding regimen and ideal duration of the fasting interval, if any, remain to be studied in relation to clinically relevant endpoints. 


\section{REFERENCES}

1. Casaer, M.P., et al., Early versus Late Parenteral Nutrition in Critically III Adults. New England Journal of Medicine, 2011. 365(6): p. 506-517.

2. Arabi, Y.M., et al., Permissive Underfeeding or Standard Enteral Feeding in Critically III Adults. N Engl J Med, 2015. 372(25): p. 2398-408.

3. Rice, T.W., et al., Randomized trial of initial trophic versus full-energy enteral nutrition in mechanically ventilated patients with acute respiratory failure. Crit Care Med, 2011. 39(5): p. 967-974 10.1097/CCM.0b013e31820a905a.

4. Allingstrup, M.J., et al., Early goal-directed nutrition versus standard of care in adult intensive care patients: the single-centre, randomised, outcome assessor-blinded EAT-ICU trial. Intensive Care Med, 2017. 43(11): p. 16371647.

5. Fivez, T., et al., Early versus Late Parenteral Nutrition in Critically III Children. N Engl J Med, 2016. 374(12): p. 1111-22.

6. Target Investigators, f.t.A.C.T.G., et al., Energy-Dense versus Routine Enteral Nutrition in the Critically III. N Engl J Med, 2018. 379(19): p. 1823-1834.

**7. de Cabo, R. and M.P. Mattson, Effects of Intermittent Fasting on Health, Aging, and Disease. N Engl J Med, 2019. 381(26): p. 2541-2551.

This review summarizes (pre)clinical evidence on intermittent fasting in health, aging and disease.

8. Gunst, J., Recovery from critical illness-induced organ failure: the role of autophagy. Crit Care, 2017. 21(1): p. 209.

9. Thiessen, S.E., G. Van den Berghe, and I. Vanhorebeek, Mitochondrial and endoplasmic reticulum dysfunction and related defense mechanisms in critical illness-induced multiple organ failure. Biochim Biophys Acta Mol Basis Dis, 2017. 1863(10 Pt B): p. 2534-2545.

10. Bear, D.E., N. Hart, and Z. Puthucheary, Continuous or intermittent feeding: pros and cons. Curr Opin Crit Care, 2018. 24(4): p. 256-261.

11. Atherton, P.J., et al., Muscle full effect after oral protein: time-dependent concordance and discordance between human muscle protein synthesis and mTORC1 signaling. Am J Clin Nutr, 2010. 92(5): p. 1080-8.

**12. McNelly, A.S., et al., Effect of Intermittent or Continuous Feed on Muscle Wasting in Critical Illness: A Phase 2 Clinical Trial. Chest, 2020. 158(1): p. 183-194.

The most recent multicentre RCT comparing continuous versus bolus feeding in critically ill patients.

13. Dirks, M.L., et al., Dietary feeding pattern does not modulate the loss of muscle mass or the decline in metabolic health during short-term bed rest. Am J Physiol Endocrinol Metab, 2019. 316(3): p. E536-E545.

14. Puthucheary, Z.A., et al., Metabolic phenotype of skeletal muscle in early critical illness. Thorax, 2018. 73(10): p. 926-935.

15. Bear, D.E. and Z.A. Puthucheary, Designing nutrition-based interventional trials for the future: addressing the known knowns. Crit Care, 2019. 23(1): p. 53. 
16. Gonzalez, J.T., et al., Intermittent versus continuous enteral nutrition attenuates increases in insulin and leptin during short-term bed rest. Eur J Appl Physiol, 2020. 120(9): p. 2083-2094.

17. Maury, E., Off the Clock: From Circadian Disruption to Metabolic Disease. Int J Mol Sci, 2019. 20(7).

18. Pickel, L. and H.K. Sung, Feeding Rhythms and the Circadian Regulation of Metabolism. Front Nutr, 2020. 7: p. 39.

19. Jamshed, H., et al., Early Time-Restricted Feeding Improves 24-Hour Glucose Levels and Affects Markers of the Circadian Clock, Aging, and Autophagy in Humans. Nutrients, 2019. 11(6).

20. Hill, A., et al., Meeting nutritional targets of critically ill patients by combined enteral and parenteral nutrition: review and rationale for the EFFORTcombo trial. Nutr Res Rev, 2020: p. 1-9.

21. Ridley, E.J., L.S. Chapple, and M.J. Chapman, Nutrition intake in the post-ICU hospitalization period. Curr Opin Clin Nutr Metab Care, 2020. 23(2): p. 111115.

22. Zhu, W., Y. Jiang, and J. Li, Intermittent versus continuous tube feeding in patients with hemorrhagic stroke: a randomized controlled clinical trial. Eur J Clin Nutr, 2020.

23. Hubbard, G.P., et al., A survey of bolus tube feeding prevalence and practice in adult patients requiring home enteral tube feeding. $\mathrm{Br} J$ Nutr, 2019. 122(11): p. 1271-1278.

24. Pletschette, Z. and J.C. Preiser, Continuous versus intermittent feeding of the critically ill: have we made progress? Curr Opin Crit Care, 2020. 26(4): p. 341345.

25. Kumar, V., et al., Age-related differences in the dose-response relationship of muscle protein synthesis to resistance exercise in young and old men. The Journal of Physiology, 2009. 587(1): p. 211-217.

*26. Waldauf, P., et al., Effects of Rehabilitation Interventions on Clinical Outcomes in Critically III Patients: Systematic Review and Meta-Analysis of Randomized Controlled Trials. Crit Care Med, 2020. 48(7): p. 1055-1065.

The most recent systematic review and meta-analysis of rehabilitation interventions in the critically ill.

27. Van Dyck, L., M.P. Casaer, and J. Gunst, Autophagy and Its Implications Against Early Full Nutrition Support in Critical Illness. Nutr Clin Pract, 2018. 33(3): p. 339-347.

28. Vanhorebeek, I., et al., Insufficient activation of autophagy allows cellular damage to accumulate in critically ill patients. . J Clin Endocrinol Metab, 2011. 96(4): p. E633-45.

29. Hermans, G., et al., Effect of tolerating macronutrient deficit on the development of intensive-care unit acquired weakness: a subanalysis of the EPaNIC trial. Lancet Respir Med, 2013. 1(8): p. 621-9.

30. Gunst, J., et al., Insufficient autophagy contributes to mitochondrial dysfunction, organ failure, and adverse outcome in an animal model of critical illness. Crit Care Med, 2013. 41(1): p. 182-94. 
31. Levine, B., M. Packer, and P. Codogno, Development of autophagy inducers in clinical medicine. J Clin Invest, 2015. 125(1): p. 14-24.

32. Di Francesco, A., et al., A time to fast. Science, 2018. 362(6416): p. 770-775.

33. Martinez-Lopez, N., et al., System-wide Benefits of Intermeal Fasting by Autophagy. Cell Metab, 2017. 26(6): p. 856-871 e5.

*34. Van Dyck, L., et al., Towards a fasting-mimicking diet for critically ill patients: the pilot randomized crossover ICU-FM-1 study. Crit Care, 2020. 24(1): p. 249.

This pilot RCT showed that 12 hours fasting induces a clear metabolic fasting response in prolonged critically ill patients.

35. Newman, J.C. and E. Verdin, beta-Hydroxybutyrate: A Signaling Metabolite. Annu Rev Nutr, 2017. 37: p. 51-76.

36. De Bruyn, A., et al., Effect of withholding early parenteral nutrition in PICU on ketogenesis as potential mediator of its outcome benefit. Crit Care, 2020. 24(1): p. 536.

37. Cox, P.J., et al., Nutritional Ketosis Alters Fuel Preference and Thereby Endurance Performance in Athletes. Cell Metab, 2016. 24(2): p. 256-68.

38. Zou, X., et al., Acetoacetate Accelerates Muscle Regeneration and Ameliorates Muscular Dystrophy in Mice. J Biol Chem, 2016. 291(5): p. 218195.

39. Goossens, C., et al., Adipose tissue protects against sepsis-induced muscle weakness in mice: from lipolysis to ketones. Crit Care, 2019. 23(1): p. 236.

40. White, $\mathrm{H}$. and B. Venkatesh, Clinical review: ketones and brain injury. Crit Care, 2011. 15(2): p. 219.

41. Stekovic, S., et al., Alternate Day Fasting Improves Physiological and Molecular Markers of Aging in Healthy, Non-obese Humans. Cell Metab, 2019. 30(3): p. 462-476 e6.

42. Goossens, C., et al., Premorbid obesity, but not nutrition, prevents critical illness-induced muscle wasting and weakness. J Cachexia Sarcopenia Muscle, 2017. 8(1): p. 89-101.

43. Bonten, M.J., et al., Intermittent enteral feeding: the influence on respiratory and digestive tract colonization in mechanically ventilated intensive-care-unit patients. Am J Respir Crit Care Med, 1996. 154(2 Pt 1): p. 394-9.

**44. Van Dyck, L. and M.P. Casaer, Intermittent or continuous feeding: any difference during the first week? Curr Opin Crit Care, 2019. 25(4): p. 356362.

Review focusing on RCTs on intermittent versus continuous feeding in critical illness.

*45. Singer, P., et al., ESPEN guideline on clinical nutrition in the intensive care unit. Clin Nutr, 2019. 38(1): p. 48-79.

The most recent ESPEN guidelines on artificial nutrition in the critically ill, which includes a systematic review on continuous versus intermittent feeding. 



\section{Key points:}

1. Increasing evidence suggests that alternating feeding and fasting periods in critically ill patients may be superior to continuous feeding.

2. Protective mechanisms include avoidance of the muscle-full effect, improvement of circadian rhythm and insulin sensitivity, and fasting-induced activation of autophagy and ketogenesis.

3. RCTs have not shown unequivocal benefit by intermittent feeding/fasting, which may be explained by the relatively small sample size and the short duration of the fasting interval.

4. Future research should investigate the ideal mode of intermittent feeding/fasting based on metabolic studies, followed by a proof-of-concept RCT that is adequately powered for clinical endpoints.

\section{Acknowledgements}

\section{Financial support and sponsorship}

JG holds a postdoctoral research fellowship supported by the University Hospitals Leuven, and C2 project funding by the KU Leuven (C24/17/070).

\section{Conflicts of interest.}

JG has no conflict of interest. ZAP has received honoraria from GlaxoSmithKline, Lyric Pharmaceuticals, Faraday Pharmaceuticals and Fresenius-Kabi; has been paid for developing and delivering educational presentations for Orion, Nutricia and Nestle; and has received non-financial support from Vitaflo. 